\title{
Multiple Reverse Direction Transmissions in IEEE 802.11 Wireless Local Area Networks
}

\author{
Raul Palacios-Trujillo ${ }^{\mathrm{a}}$, Jesus Alonso-Zarate ${ }^{\mathrm{b}}$, Nelson L. S. da Fonseca ${ }^{\mathrm{a}}$, and Fabrizio Granelli ${ }^{\mathrm{c}}$ \\ anstitute of Computing, University of Campinas, Brazil, \{rpalacios, nfonseca\}@ic.unicamp.br \\ ${ }^{\mathrm{b}}$ Centre Tecnològic de Telecomunicacions de Catalunya (CTTC), Spain, jesus.alonso@cttc.es \\ ${ }^{c}$ Department of Information Engineering and Computer Science, University of Trento, Italy, granelli@disi.unitn.it
}

\begin{abstract}
This paper proposes a new Reverse Direction (RD) Medium Access Control (MAC) protocol to improve the throughput and energy efficiency of IEEE 802.11 Wireless Local Area Networks (WLANs). The proposed protocol allows a source device to transmit a burst of data packets to the intended destination device in a single channel access opportunity. After the successful reception of each data packet, the destination device may opportunistically respond with a data packet, thus being able to perform multiple RD transmissions. This operation can reduce the overall channel access overhead, hence increasing the efficiency of data transmission between two sender-receiver devices. The results obtained by means of theoretical analysis and computer-based simulation show that the novel RD protocol can outperform existing IEEE 802.11 protocols by yielding gains close to $60 \%$. $^{*}$
\end{abstract}

\section{INTRODUCTION}

The IEEE $802.11 \mathrm{n}$ amendment of the IEEE 802.11 Standard introduced various advanced mechanisms to achieve high throughput and energy efficiency in IEEE 802.11 WLANs [1]. One of the proposed mechanisms is the Reverse Direction Protocol (RDP) included in the MAC layer. This mechanism represents an extension of the existing IEEE 802.11 contentionbased MAC protocols, namely: the Distributed Coordination Function (DCF) and the IEEE 802.11e Enhanced Distributed Channel Access (EDCA). DCF is the basic channel access method based on Carrier Sense Multiple Access with Collision Avoidance (CSMA/CA) and a Binary Exponential Backoff (BEB) algorithm. In addition, EDCA is an extension of DCF to support prioritized channel access and occupancy time for different Quality of Service (QoS) traffic categories.

In both DCF and EDCA, the wireless station (STA) that wins the contention gains access to the channel for a reserved period of time to transmit data to one arbitrary destination. This period of time is referred to as Transmission Opportunity (TXOP) and only permits (unidirectional) data transmissions from the STA that seizes the channel to the intended destination, i.e. in the forward direction. However, with RDP, once the STA holding a TXOP has sent its data, it may grant permission to the destination for an immediate data response during the unused part of its TXOP. This operation may allow

* This work was funded by the GREENET European research project under grant PITN-GA-2010-264759, the CNPq Brazilian research agency, and Motorola Brazil. (bidirectional) data transmissions in both the forward and reverse (from the destination to the transmitting STA) directions during a TXOP. Therefore, RDP can provide performance improvement gains for certain traffic patterns by reducing the overall channel access overhead.

The use of reverse direction (or bidirectional) transmissions in WLANs was originally proposed in [2], before RDP was included in the Standard. Since then, several papers [3]-[8], have introduced similar approaches with different purposes. Existing RD protocols can be classified into two categories: (i) proactive, where the RD exchange sequence is initiated by the transmitter, or (ii) reactive, where the RD exchange sequence is initiated by the receiver. More specifically, proactive RD protocols [3], [4] are based on the operation of RDP. Once a transmitter has obtained a TXOP, it may send an RD Grant (RDG) to the receiver to allocate the remaining time of its TXOP for RD data transfer. However, the unused TXOP duration may be insufficient for the receiver to send all its data back. In that case, the receiver may need to request and wait for new RDGs from the transmitter or gain its own TXOPs.

On the other hand, reactive RD protocols [2], [5]-[8] extend the operation of DCF as follows. Upon reception of valid data, the receiver may reserve the channel by extending the occupancy time to perform a contention-free data transmission back to the transmitter. This kind of RD protocols can be more suitable in some scenarios due to their ability to adapt to the actual traffic requirements of a network. Particularly, the feasibility of reactive RD exchange operation for infrastructure WLANs was investigated in [7] and in our previous work [8]. The results presented in these papers show that reactive RD approaches can outperform DCF when bidirectional data flows exist between the Access Point (AP) and the STAs. In the long term, DCF provides an equal opportunity to access the channel for all contenting STAs, including the AP. However, the AP should be provided with a larger share of the channel access opportunities since it carries data destined to all the STAs. This is possible by allowing the AP to dynamically initiate RD exchanges when receiving data from the STAs, thus improving the overall network performance.

The reactive RD protocols proposed in [7] and [8] were designed to allow the receiver to transmit a single data packet to the transmitter in response to a received data packet. However, in some cases there may exist more data packets ready 
to be transmitted in both directions. As a , the opportunities for exchanging more information during channel access are missed, since such protocols do not leverage the potential of multiple bidirectional transmissions. This is the motivation for the new standard-compliant RD protocol introduced in this paper.

Based on our previous BidMAC design [8], the proposed MR-BidMAC protocol combines burst transmissions using the IEEE 802.11e TXOP operation and multiple reactive RD exchanges. Essentially, the transmitter can send a burst of data packets to the intended receiver in each channel access opportunity. After valid reception of each data packet, the receiver may opportunistically respond with a data packet, initiating multiple rounds of RD transmissions. This extended operation can increase the achievable throughput and energy efficiency of BidMAC.

In this paper, we present an analytical model to compute the maximum achievable throughput and energy efficiency of the novel MR-BidMAC protocol. By means of the proposed analytical model and computer-based simulations, we provide a comprehensive performance evaluation of the protocol. We compare the performance of MR-BidMAC with that of DCF integrating burst transmissions (hereafter referred to as MRDCF) and BidMAC.

The remainder of this paper is structured as follows. The new MR-BidMAC protocol is introduced in Section II. Section III includes the theoretical analysis of the throughput and energy efficiency of the proposed protocol. The performance evaluation of the protocol is presented in Section IV. Finally, Section V draws conclusions by summarizing the most relevant results presented in the paper.

\section{New ReACtive RD MAC PROTOCOL}

In this section, we introduce a novel reactive (i.e., receiverinitiated) RD MAC protocol, called MR-BidMAC, to increase the throughput and energy efficiency of IEEE 802.11 WLANs.

\section{A. Protocol Description}

MR-BidMAC is a standard-compliant protocol that is based on the combination of two standard mechanisms implemented on top of the legacy DCF procedure. One is the IEEE 802.11e TXOP technique to allow burst transmissions. The other is the reactive operation of the IEEE 802.11n RDP scheme to enable receiver-initiated RD exchanges. In other words, the new protocol represents an extension of the BidMAC protocol [8] integrating the TXOP operation to allow multiple rounds of bidirectional transmissions.

When the AP and the STAs have data to transmit, they contend for access to the channel using the rules of DCF. This means that they wait until the channel is sensed idle during a DCF Interframe Space (DIFS) period, or Extended Interframe Space (EIFS) at the end of a collision. After a DIFS or EIFS period, they access the channel following exponential backoff rules based on a Contention Window (CW) to generate random backoff times. Whenever one of them obtains the channel, it may directly send the data to the intended destination, indicating the expected transmission duration in transmitted data packets. Alternatively, before the data transmission, it may transmit a Request-To-Send (RTS) packet to the destination and wait to receive a Clear-To-Send (CTS) packet after a Short Interframe Space (SIFS) period. This handshake allows combating the hidden terminal problem and reducing the collision times.

Once the sender, either the AP or an STA, gains access to the channel, it can transmit data to the intended receiver for a reserved period of time (i.e. a TXOP). During a TXOP, the sender may initiate a burst transmission in which several data packets can be transmitted to the receiver (up to a maximum allowed number). Each successful reception of a data packet is followed by a positive Acknowledgment (ACK) response from the receiver after a SIFS period. In addition, with the reactive $\mathrm{RD}$ approach, the receiver may respond to the sender with a data packet, thus producing one or more receiver-initiated bidirectional data exchanges.

More specifically, the receiver may send a data packet with a piggybacked ACK addressed to the transmitter of the received data packet. The data packet can be of arbitrary length up to the maximum allowed byte length of payload. The transmission rate of the data packet is kept constant for both the forward and reverse direction transmissions. Note that the rate could be reduced for the RD transmission to increase the probability of successful transmission under bad channel conditions in the reverse channel. Also, the value of the duration field in the transmitted data packet is extended to reserve the channel for the duration to complete each bidirectional data exchange. The reserved time accounts for both forward and backward data transmissions and the terminating ACK transmission from the transmitter.

The use of multiple bidirectional transmissions can further improve the network throughput and energy efficiency, compared to the case where a single bidirectional exchange is allowed. In order to increase the opportunities for multiple bidirectional transmissions, the AP and the STAs may hold data packets for a given time (referred to as holding time). They may wait for a shorter time than the holding time provided that they reach the maximum allowed duration of a burst transmission before the holding time expires. In this case, they may immediately attempt access to the channel to send the burst of data packets to the intended destination.

The operation of MR-BidMAC could be extended to support packet aggregation and block ACK, which are features defined in the IEEE 802.11 Standard.

\section{B. Example of Operation}

The operation of MR-BidMAC is exemplified in Fig. 1. In this example, $\mathrm{STA}_{1}$ and the AP exchange several data packets using the RTS/CTS mechanism while other STAs overhear the data exchange. The energy profiles of $\mathrm{STA}_{1}$, the AP, and other STAs when transmitting, receiving (or overhearing), and listening to an idle channel are shown in Fig. 1.

After a DIFS period and a random backoff time, $\mathrm{STA}_{1}$ is the first to seize the channel by transmitting an RTS packet 


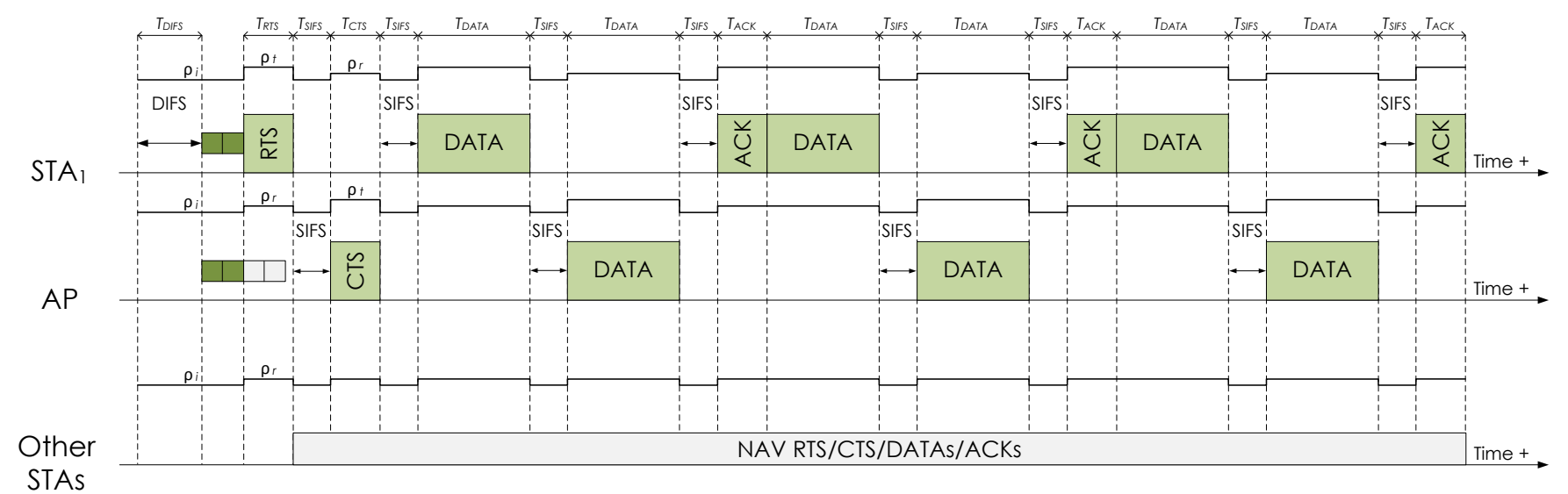

Fig. 1. Example of operation of the proposed MR-BidMAC protocol. After the RTS/CTS handshake, $\mathrm{STA}_{1}$ and the AP perform various rounds of bidirectional data transmissions within a single channel access, while other STAs overhear the data exchange. The energy profiles of STA 1 , the AP, and other STAs during transmission, reception (or overhearing), and idle channel listening are shown in the figure.

to the AP. Following a SIFS period, the AP responds with a CTS packet that includes an extended duration to account for the multiple rounds of bidirectional transmissions. Upon successful reception of the CTS packet, $\mathrm{STA}_{1}$ transmits a data packet to the AP, which sends back a data packet after a SIFS period. The newly received data packet can be interpreted by $\mathrm{STA}_{1}$ as an implicit ACK for its transmitted data packet. After a SIFS period, $\mathrm{STA}_{1}$ replies with an explicit ACK packet to complete the first bidirectional data exchange. Then, it transmits a new data packet to initiate the second data exchange. The procedure described above repeats until the third data exchange concludes with the transmission of an ACK packet from $\mathrm{STA}_{1}$. During the bidirectional data transfer, other STAs update their Network Allocation Vectors (NAVs) with the duration value contained in overheard control and data packets. They will not attempt access to the channel for the NAV duration.

\section{TheoreticAl AnALysis}

The maximum achievable throughput and energy efficiency of MR-BidMAC is analyzed in this section. This analysis is based on the analytical model presented in [9] and its extension reported in [10] to evaluate the saturation throughput of an IEEE 802.11 DCF network.

\section{A. System Model and Assumptions}

A Basic Service Set (BSS) composed of an AP and $n$ associated STAs in the Basic Service Area (BSA) is considered. All devices are equipped with IEEE 802.11 interfaces enabling a single antenna for communications, hence forming a SingleInput Single-Output (SISO) communications system. Wireless communication within the BSS occurs between the AP and the STAs using a shared radio channel. The size of the BSA allows all the STAs of the BSS to overhear the transmissions between each STA and the AP in both directions, thus creating a single-hop network with no hidden terminals. Note that the AP can deliver data to any STA of the BSS.

In order to compute the upper bound performance of MRBidMAC, it is assumed that the considered network operates in saturation conditions. This means that the AP and all the
STAs always have data packets in their transmission queues. To transmit data, the AP and the STAs compete for access to the channel. In any transmission cycle, they perform carrier sensing and execute the random backoff procedure. Once one of them seizes the channel, it performs an RTS/CTS handshake to send one or several data packets to the intended receiver. All data packets have constant byte length (no fragmentation needed). It is assumed that no packet error occurs due to channel variations and there exists no capture effect. In addition, no management packets, such as beacons and association requests, are transmitted.

\section{B. System Parameters}

The duration of the SIFS, DIFS, and EIFS periods are denoted by $T_{S I F S}, T_{D I F S}$, and $T_{E I F S}$, respectively. The minimum value of the $\mathrm{CW}$ size is represented by $C W_{\text {min }}$, whereas $C W_{\max }$ corresponds to the maximum $\mathrm{CW}$ size. The transmission times of RTS, CTS, data, and ACK packets are expressed as $T_{R T S}, T_{C T S}, T_{D A T A}$, and $T_{A C K}$, respectively. The propagation delay is referred to as $\delta$. Let $\rho_{t}, \rho_{r}$, and $\rho_{i}$ be the power consumed by the radio interface of a device when transmitting, receiving or overhearing, and idle channel listening, respectively.

\section{Throughput}

The throughput $S$ is defined as the fraction of time that the channel is used to successfully transmit payload bits. Therefore, based on [9] and [10], the saturation throughput of the network can be expressed as

$$
S=\frac{\alpha P_{t r} P_{s} E[P]^{\prime}}{\left(1-P_{t r}\right) \sigma+P_{t r} P_{s} T_{s}^{\prime}+P_{t r}\left(1-P_{s}\right) T_{c}^{\prime}}
$$

where

- $\alpha$ : a new variable that we add to represent the number of successful data transmissions within a given slot time. Note that $\alpha$ may be different according to the considered MAC protocol. For example, in DCF the AP or an STA can transmit a data packet in each channel access, thus $\alpha=1$. 
- $P_{t r} P_{s}$ : probability of successful transmission in a given slot time. These variables are defined in [9] and represent what can happen in a randomly chosen slot time, namely:

- $P_{t r}$ : refers to the probability that there is at least one transmission in the considered slot time, expressed as (10) in [9].

- $P_{s}$ : denotes the probability that a transmission occurring in the channel is successful. It is given by the probability that only one STA transmits in the channel, provided that at least one STA transmits, written as (11) in [9].

- $E[P]^{\prime}$ : average packet payload size considering the extension in [10] to more accurately model the backoff freezing operation, given by (10) in [10].

- $1-P_{t r}$ : probability that a given slot time is empty.

- $P_{t r}\left(1-P_{s}\right)$ : probability that a collision occurs in a given slot time.

- $\sigma$ : duration of an empty slot time.

- $T_{s}^{\prime}$ : duration of a successful transmission considering the backoff freezing modification and the additional backoff slot $\sigma$ after a DIFS period for a listening STA that will decrement its backoff counter by one unit [10], which is computed as

$$
T_{s}^{\prime}=\frac{T_{s}}{1-B_{0}}+\sigma
$$

where $B_{0}$ refers to the probability that a successfully transmitting STA may access the channel in the first slot after a DIFS period. This occurs when an STA extracts a new backoff counter value equal to zero, i.e. with probability $B_{0}=\frac{1}{W}$. W is defined for convenience as $W=C W_{\min }+1$. The reason is that initially the backoff counter value randomly chosen by a contending STA may range from 0 to $C W_{\min }$. This leads to a $\mathrm{CW}$ size of $W$ possible values. Note that $T_{s}$ will vary depending on the analyzed MAC protocol. For instance, $T_{s}$ for DCF with RTS/CTS is written as (17) in [9].

- $T_{c}^{\prime}$ : duration of a collision considering the updated model and the EIFS period [10], expressed as

$$
T_{c}^{\prime}=T_{c}+\sigma
$$

where $T_{c}=T_{R T S}+\delta+T_{E I F S}$.

The successful transmission duration of MR-BidMAC in saturation conditions $\left(T_{s}\right)$ is shown in Fig. 1. It consists of a DIFS period, an RTS transmission plus the propagation delay, a SIFS period, a CTS transmission plus the propagation delay, a SIFS period, and a sequence of bidirectional data transmissions between sender and receiver. This sequence includes $2 \cdot \beta$ data transmissions plus $2 \cdot \beta$ propagation delays, $2 \cdot(1+\beta)$ SIFS periods, and $\beta$ ACK transmissions plus $\beta$ propagation delays. The $\beta$ value determines the maximum number of transmitted data packets by each device of a pair of sender and receiver in a single channel access opportunity. As a result, $T_{s}$ for MR-BidMAC is written as

$$
\begin{aligned}
T_{s} & =T_{R T S}+T_{C T S}+\beta\left(2 \cdot T_{D A T A}+T_{A C K}\right)+T_{D I F S} \\
& +2 \cdot(1+\beta) T_{S I F S}+(2+3 \cdot \beta) \delta
\end{aligned}
$$

Since MR-BidMAC involves the transmission of two data packets in each bidirectional transfer, $\alpha$ is equal to $2 \cdot \beta$. Hence, the saturation throughput of MR-BidMAC from a network perspective is obtained by (1) using (10) and (11) in [9], (10) in [10], and (2)-(4).

\section{Energy Efficiency}

The energy efficiency $\eta$ is defined as the amount of energy consumed during the fraction of time that the channel is used to successfully transmit payload bits. Considering the expression of $S$, the network energy efficiency can be similarly formulated as

$$
\eta=\frac{\alpha P_{t r} P_{s} E[P]^{\prime}}{\left(1-P_{t r}\right) E_{\sigma}+P_{t r} P_{s} E_{s}^{\prime}+P_{t r}\left(1-P_{s}\right) E_{c}^{\prime}}
$$

where

- $E_{\sigma}$ : energy consumed during an empty slot time, that is

$$
E_{\sigma}=\sigma(n+1) \rho_{i}
$$

where all devices consume energy for being idle during a slot time $\sigma$.

- $E_{s}^{\prime}$ : energy consumed during a successful transmission considering the updated model [10], computed as

$$
E_{s}^{\prime}=\frac{E_{s}}{1-B_{0}}+\sigma(n+1) \rho_{i}
$$

Note that $E_{s}$ will change depending on the MAC protocol under analysis.

- $E_{c}^{\prime}$ : energy consumed during a collision taking into account the modifications of [10] and the EIFS period, which is given as

$$
\begin{aligned}
& E_{c}^{\prime}=E_{c}+\sigma(n+1) \rho_{i} \\
& E_{c}=E_{t}+E_{r}+E_{i}\left\{\begin{array}{l}
E_{t}=T_{R T S} E[k] \rho_{t} \\
E_{r}=T_{R T S}(n+1-E[k]) \rho_{r} \\
E_{i}=\left(T_{E I F S}+\delta\right)(n+1) \rho_{i}
\end{array}\right.
\end{aligned}
$$

where $E[k]$ is the average number of devices involved in a collision (including the AP and the $n$ STAs). Note that in a collision $E[k]$ devices consume energy to transmit the RTS packets $\left(E_{t}\right)$ whereas the rest of devices consume energy to overhear the collision of the RTS packets $\left(E_{r}\right)$. All devices consume energy for being idle during an EIFS period, the propagation delay of the RTS transmission, and the additional slot time $\left(E_{i}\right)$. To compute $E[k]$, the Bayesian theorem is used. The average number of devices involved in a collision is given by the summation of the probabilities that two or more $(m)$ devices up to $n+1$ devices (considering all possible combinations) cause a collision conditioned that there is a collision in a given slot. Thus, $E[k]$ is expressed as

$$
E[k]=\frac{\sum_{m=2}^{n+1}\left(\begin{array}{c}
n+1 \\
m
\end{array}\right) \tau^{m}(1-\tau)^{n+1-m}}{P_{t r}\left(1-P_{s}\right)}
$$

The energy consumption of MR-BidMAC during a successful bidirectional transmission $\left(E_{s}\right)$ is shown in Fig. 1. The 
transmitter consumes energy to transmit the RTS, $\beta$ data, and $\beta$ ACK packets to the receiver whereas the receiver consumes energy to transmit the CTS and $\beta$ data packets to the transmitter $\left(E_{t}\right)$. The transmitter consumes energy to receive the CTS and $\beta$ data packets from the receiver whereas the receiver consumes energy to receive the RTS, $\beta$ data, and $\beta$ ACK packets from the transmitter $\left(E_{r}\right) . n-1$ STAs (one of the $n$ STAs acts as the transmitter or the receiver) consume energy to overhear the RTS, CTS, $2 \cdot \beta$ data, and $\beta$ ACK transmissions $\left(E_{r}\right)$. Also, the AP and all the STAs consume energy for being idle during the DIFS period, $2 \cdot(1+\beta)$ SIFS periods, and $2+3 \cdot \beta$ propagation delays interleaving the transmissions $\left(E_{i}\right)$. Consequently, $E_{s}$ for MR-BidMAC is expressed as

$$
\begin{aligned}
& E_{s}=E_{t}+E_{r}+E_{i} \\
& E_{t}=\left(T_{R T S}+T_{C T S}+\beta \cdot\left(2 \cdot T_{D A T A}+T_{A C K}\right)\right) \rho_{t} \\
& E_{r}=\left(T_{R T S}+T_{C T S}+\beta \cdot\left(2 \cdot T_{D A T A}+T_{A C K}\right)\right) n \rho_{r} \\
& E_{i}=\left(T_{D I F S}+2 \cdot(1+\beta) T_{S I F S}+(2+3 \cdot \beta) \delta\right)(n+1) \rho_{i}
\end{aligned}
$$

The network energy efficiency of MR-BidMAC in saturation conditions is given by (5) using $\alpha=2 \cdot \beta$, (10) and (11) in [9], (10) in [10], and (6)-(10).

\section{Performance Evaluation}

A comprehensive performance evaluation of MR-BidMAC is presented in this section. The analysis presented in the previous section was validated by means of computer-based simulations. The effects of different values of the traffic load, packet length, and $\beta$ parameter on the performance of MRBidMAC were studied. All results were compared to the performance of DCF, MR-DCF (DCF with burst transmissions), and BidMAC.

\section{A. Simulation Scenario and Setup}

The operation rules of the evaluated protocols were implemented in a custom-made object-oriented link-level Python simulator. The simulation scenario was implemented according to the description of the system model and assumptions presented in the previous section. In the simulator, the AP and each STA in the network constitute different entities (instances of a class) that execute the code that would be implemented in a real platform. They generate data packets of constant length following a Poisson arrival distribution, i.e. packets are generated on average at a given rate but the packet generation time is random. All the STAs generate data packets addressed to the AP at an equal rate whereas the AP generates as many data packets as all the STAs on average. The destination of each data packet transmitted by the AP is randomly selected among all the STAs of the network with equal probability.

All the protocols implemented in the simulator enable the RTS/CTS handshake, burst transmission, and a holding time. As explained in previous sections, this time is used to hold data packets ready to be transmitted in order to increase the opportunities for multiple data transmissions during channel access. The operation rules of all the protocols were simulated following the specifications provided in previous sections.
All simulation results presented in this section were derived using 10 replications and with a duration of each simulation of $15 \mathrm{~s}$. Confidence intervals with a confidence level of $95 \%$ and obtained by the method of replication were employed. The width of the confidence intervals was at most $2 \%$ of the mean value. Therefore, they are omitted in the figures for the sake of visualization.

\section{B. System Parameters}

The system parameters corresponding to the IEEE 802.11n specification were used to compute the analytical and simulation results. This amendment of the Standard specifies various Physical (PHY) layer techniques. Among them, the Extended Rate PHY (ERP) specification with Orthogonal Frequency Division Multiplexing (OFDM) modulation for SISO communications was selected. This PHY mode provides 8 transmission rates ranging from 6 to $54 \mathrm{Mbps}$ with Number of Data Bits Per OFDM Symbol $\left(N_{D B P S}\right)$ from 24 to 216, respectively. Note that RTS and data transmissions can be performed using any of these rates. However, CTS and ACK packets must be transmitted at the basic rates 6,12 , or 24 Mbps, in accordance with the basic rate selection rules in [1].

The expression to compute the transmission time of each packet using the ERP-OFDM PHY mode is given in [1] as

$$
T_{x}=T_{\text {pre }}+T_{\text {sig }}+T_{\text {sym }}\left\lceil\frac{L_{\text {serv }}+8 \cdot L_{x}+L_{\text {tail }}}{N_{D B P S}}\right\rceil+T_{\text {sigEx }}
$$

where $x$ is the packet type and all the variables and their values are provided in Table I. The MAC packet length is referred to as $L_{x}$. A data packet or MAC Protocol Data Unit (MPDU) includes the frame body or MAC Service Data Unit (MSDU) together with a MAC header $\left(L_{M A C h d r}\right)$ and a Frame Check Sequence (FCS), $L_{F C S}$. For instance, for an MSDU of 1500 bytes and RTS/data and CTS/ACK transmission rates of 54 and $24 \mathrm{Mbps}$, respectively, $T_{R T S}, T_{C T S}, T_{D A T A}$, and $T_{A C K}$ are obtained by (11) as 30, 34, 254, and $34 \mu s$, respectively. Note that the propagation delay following a packet transmission $(\delta)$ is neglected because an ideal channel was considered to evaluate the performance of the protocols.

Table I also includes other variables that were obtained as follows. The DIFS period was computed by [1] as $T_{D I F S}=T_{S I F S}+2 \cdot \sigma$ and the EIFS period as $T_{E I F S}=T_{D I F S}+T_{S I F S}+T_{A C K}(6 M b p s)$. A holding time of $100 \mathrm{~ms}$ was considered to run simulations since it produced the best performance results for all the mechanisms when multiple data transmissions were enabled. The values of power consumption in transmission, reception, and idle channel listening states were taken from [11].

\section{Results}

For all the figures presented in this section, the solid lines refer to the analytical results whereas the markers are related to the simulation results. The results presented in the figures were plotted for a WLAN composed of an AP and 20 STAs, an MSDU length of 1500 bytes, and PHY control and data rates of 24 and $54 \mathrm{Mbps}$, respectively. 
TABLE I

SYSTEM PARAMETERS

\begin{tabular}{|c|c|}
\hline Parameter & Value \\
\hline Slot time $(\sigma)$ & $9 \mu \mathrm{s}$ \\
\hline SIFS period $\left(T_{S I F S}\right)$ & $10 \mu \mathrm{s}$ \\
\hline DIFS period $\left(T_{D I F S}\right)$ & $28 \mu \mathrm{s}$ \\
\hline EIFS period $\left(T_{E I F S}\right)$ & $88 \mu s$ \\
\hline Minimum CW size $\left(C W_{\min }\right)$ & 15 \\
\hline Maximum CW size $\left(C W_{\max }\right)$ & 1023 \\
\hline Preamble time $\left(T_{\text {pre }}\right)$ & $16 \mu \mathrm{s}$ \\
\hline Signal time $\left(T_{\text {sig }}\right)$ & $4 \mu \mathrm{s}$ \\
\hline OFDM symbol period $\left(T_{s y m}\right)$ & $4 \mu \mathrm{s}$ \\
\hline Signal extension period $\left(T_{\text {sigEx }}\right)$ & $6 \mu \mathrm{s}$ \\
\hline Service bits $\left(L_{\text {serv }}\right)$ & $16 \mathrm{~b}$ \\
\hline Tail bits $\left(L_{t a i l}\right)$ & $6 \mathrm{~b}$ \\
\hline Length of RTS $\left(L_{R T S}\right)$ & $20 \mathrm{~B}$ \\
\hline Length of CTS/ACK $\left(L_{C T S}=L_{A C K}\right)$ & $14 \mathrm{~B}$ \\
\hline Length of the MAC header $\left(L_{M A C h d r}\right)$ & $30 \mathrm{~B}$ \\
\hline Length of FCS $\left(L_{F C S}\right)$ & $4 \mathrm{~B}$ \\
\hline Transmission power consumption $\left(\rho_{t}\right)$ & $1.65 \mathrm{~W}$ \\
\hline Reception power consumption $\left(\rho_{r}\right)$ & $1.4 \mathrm{~W}$ \\
\hline Idle power consumption $\left(\rho_{i}\right)$ & $1.15 \mathrm{~W}$ \\
\hline Holding time & $100 \mathrm{~ms}$ \\
\hline
\end{tabular}

In Fig. 2, we evaluate the influence of $\beta=1$ and $\beta=3$ rounds of data transmissions on the performance of the protocols with increasing traffic loads. More specifically, the network throughput and energy efficiency of the evaluated protocols versus the total offered traffic load are shown in Figs. 2a and $2 \mathrm{~b}$, respectively. It can be seen that both MR-DCF and MRBidMAC with $\beta=3$ outperform DCF and BidMAC (i.e. both MR-DCF and MR-BidMAC with $\beta=1$ ), respectively, for high traffic loads. The reason is that, as more data packets are ready for transmission, more opportunities for multiple data transmissions emerge during channel access. Furthermore, MRBidMAC with $\beta=3$ achieves the highest performance since it allows up to three bidirectional data exchanges between the AP and an STA in a single channel access opportunity. However, the maximum performance gain of MR-BidMAC versus MRDCF is obtained with $\beta=1$ due to the higher influence of the reduced channel access overhead for data transmission. Therefore, the throughput gains are up to $29 \%$ for $\beta=1$ and $17 \%$ for $\beta=3$. Likewise, the energy efficiency gains are up to $27 \%$ for $\beta=1$ and $16 \%$ for $\beta=3$.

The impact of variable MSDU length on the saturation performance of the evaluated protocols is analyzed in Fig. 3. The saturation network throughput is shown in Fig. 3a and the saturation network energy efficiency in Fig. 3b. We can observe that the saturation performance of the evaluated protocols increases as the MSDU length is longer since more information is contained in each transmitted data packet. It can also be seen that MR-BidMAC outperforms MR-DCF for all the MSDU lengths with both $\beta=1$ and $\beta=3$ rounds of data transmissions. However, although the performance of MR-BidMAC is higher with $\beta=3$, a higher performance gain versus MR-DCF is obtained with $\beta=1$. The reason was previously explained to understand the performance behavior of MR-BidMAC versus the traffic load. In addition, the gain decreases as the MSDU length increases for a similar reason. In this case, longer data packets increase the data transmission time during channel access, hence reducing the influence of

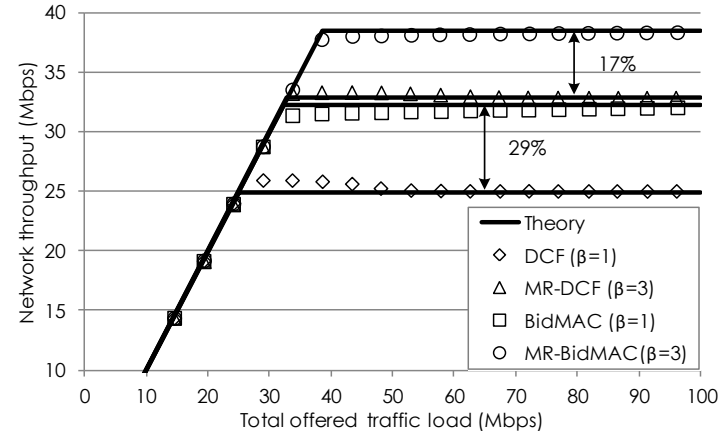

(a) Throughput

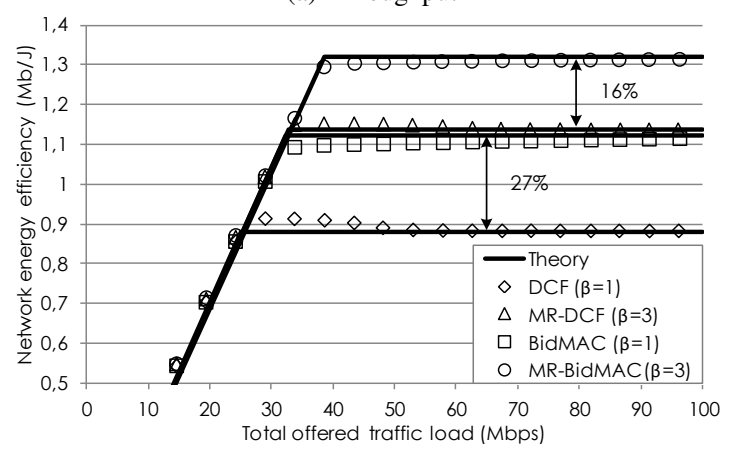

(b) Energy efficiency

Fig. 2. Network throughput and energy efficiency of the evaluated protocols with $\beta=1$ and $\beta=3$ rounds of data transmissions versus the traffic load.

the channel access overhead. Thus, the throughput gains range from $68 \%$ to $22 \%$ for $\beta=1$ and from $54 \%$ to $13 \%$ for $\beta=3$. Similarly, the energy efficiency gains range from $66 \%$ to $21 \%$ for $\beta=1$ and from $53 \%$ to $12 \%$ for $\beta=3$.

We study the impact of variable $\beta$-rounds of data transmissions on the saturation performance of the protocols from $\beta=1$ to $\beta=10$ in Fig. 4. In particular, the saturation network throughput and energy efficiency of the evaluated protocols are presented in Figs. $4 \mathrm{a}$ and $4 \mathrm{~b}$, respectively. It can be seen that the saturation performance of the protocols increases as the value of $\beta$ increases, since more data packets can be transmitted in each channel access opportunity. The maximum saturation performance of the protocols is obtained for a $\beta=10$ value, although the performance slightly increases after a $\beta=5$ value. The reason is that, in a single channel access opportunity, the contribution of the channel access overhead to the total transmission time becomes marginal with respect to the data transmission time. This fact is even more noticeable for MR-BidMAC due to the use of bidirectional transmissions, as explained earlier for Figs. 2 and 3. For this reason, we can observe that the maximum gains of MR-DCF between $\beta=1$ and $\beta=10$ are up to $48 \%$ and $44 \%$ in terms of saturation network throughput and energy efficiency, respectively. In contrast, those for MR-BidMAC are lower, up to $28 \%$ and $26 \%$, respectively.

In addition, we can see in Figs. $4 \mathrm{a}$ and $4 \mathrm{~b}$ that MR-BidMAC always achieves the highest performance for all the evaluated values of $\beta$. The throughput gains versus MR-DCF range from $29 \%$ to $12 \%$ whereas the energy efficiency gains vary between $27 \%$ and $11 \%$. 


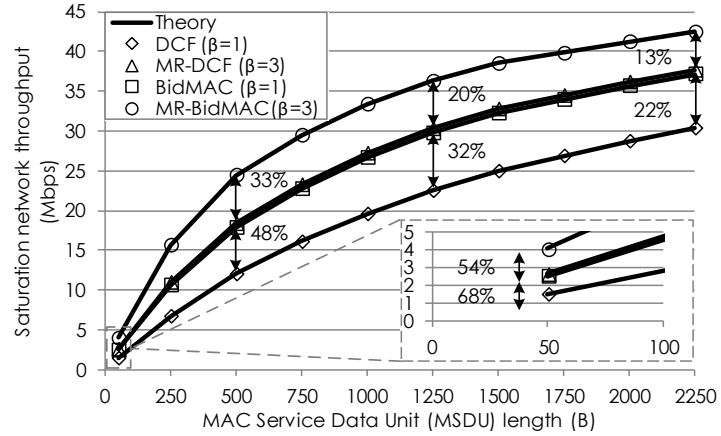

(a) Throughput

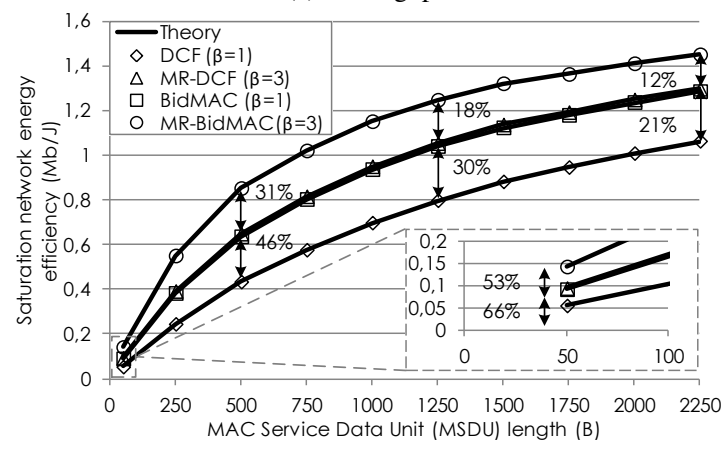

(b) Energy efficiency

Fig. 3. Saturation network throughput and energy efficiency of the protocols with $\beta=1$ and $\beta=3$ rounds of data transmissions versus the MSDU length.

To conclude, the results presented in this section show that the use of multiple data transmissions during channel access can help improve the performance of DCF (i.e. MR-DCF). Furthermore, the use of multiple bidirectional transmissions in each channel access opportunity (i.e. MR-BidMAC) represents a promising solution to significantly increase the throughput and energy efficiency of IEEE 802.11 WLANs.

\section{Conclusions}

A novel standard-compliant RD protocol for highthroughput energy-efficient IEEE 802.11 WLANs, called MRBidMAC, was presented in this paper. MR-BidMAC allows multiple $(\beta)$ rounds of receiver-initiated bidirectional data exchanges between a pair of sender-receiver devices in a single channel access opportunity. The throughput and energy efficiency of MR-BidMAC were evaluated via theoretical analysis and computer-based simulations in a WLAN composed of an $\mathrm{AP}$ and a finite number of STAs. The performance of MRBidMAC was compared to that of the IEEE 802.11 DCF protocol integrating multiple $\beta$-rounds of data transmissions (called MR-DCF in the paper) and an existing RD protocol called BidMAC. The results presented in this paper show that MR-BidMAC can achieve a performance improvement of up to $54 \%$ versus MR-DCF (with $\beta=3$ ) for scenarios with heavy traffic and small data packets. In addition, the saturation performance of MR-BidMAC can increase up to $28 \%$ as the value of $\beta$ increases from $\beta=1$ (BidMAC) to $\beta=10$.

In future work, we will evaluate the performance of MRBidMAC in scenarios with different classes of traffic and also

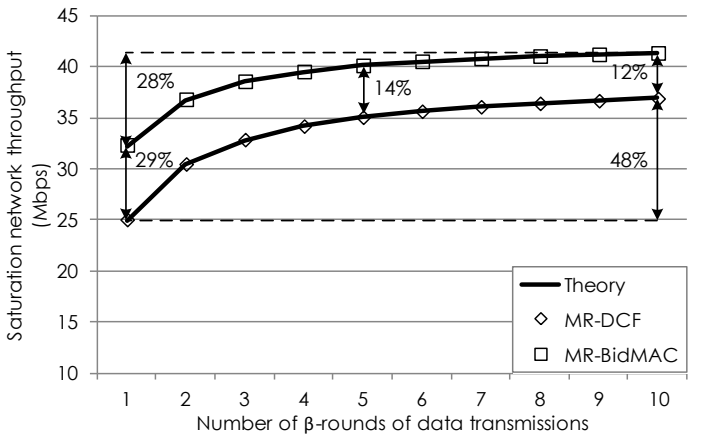

(a) Throughput

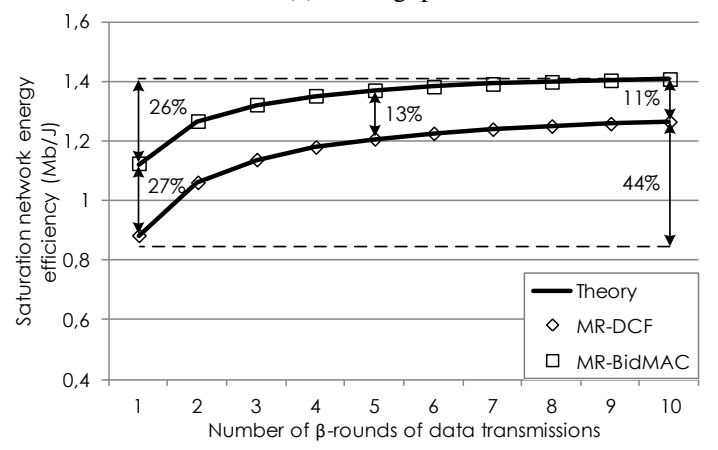

(b) Energy efficiency

Fig. 4. Saturation network throughput and energy efficiency of the evaluated protocols versus the number of $\beta$-rounds of data transmissions.

extend its operation to support packet aggregation and block ACK. Also, we will test the protocol in real-life environments through programmable wireless platforms.

\section{REFERENCES}

[1] IEEE, Part 11: Wireless LAN Medium Access Control (MAC) and Physical Layer (PHY) Specifications, IEEE 802.11 Std., 2012.

[2] H. Wu, Y. Peng, K. Long, S. Cheng, and J. Ma, "Performance of Reliable Transport Protocol over IEEE 802.11 Wireless LAN: Analysis and Enhancement," in IEEE INFOCOM 2002, vol. 2, Jun. 2002, pp. 599-607.

[3] M. Ozdemir, G. Daqing, A. B. McDonald, and J. Zhang, "Enhancing MAC Performance with a Reverse Direction Protocol for High-Capacity Wireless LANs," in IEEE VTC 2006, Sep. 2006, pp. 1-5.

[4] D. Akhmetov, "802.11n: Performance Results of Reverse Direction Data Flow," in IEEE PIMRC 2006, Sep. 2006, pp. 1-3.

[5] D.-H. Kwon, W.-J. Kim, and Y.-J. Suh, "A Bidirectional Data Transfer Protocol for Capacity and Throughput Enhancements in Multi-rate Wireless LANs," in IEEE VTC 2004, vol. 4, Sep. 2004, pp. 3055-3059.

[6] W. Choi, J. Han, B. J. Park, and J. Hong, "BCTMA (Bi-directional CutThrough Medium Access) Protocol for 802.11-based Multi-hop Wireless Networks," in ACM ISSADS 2005, Jan. 2005, pp. 377-387.

[7] N. S. P. Nandiraju, H. Gossain, D. Cavalcanti, K. R. Chowdhury, and D. P. Agrawal, "Achieving Fairness in Wireless LANs by Enhanced IEEE 802.11 DCF," in IEEE WiMob 2006, Jun. 2006, pp. 132-139.

[8] R. Palacios, F. Franch, F. Vazquez-Gallego, J. Alonso-Zarate, and F. Granelli, "Experimental Evaluation of Reverse Direction Transmissions in WLAN Using the WARP Platform," in IEEE ICC, 2015, pp. 6139-6145.

[9] G. Bianchi, "Performance Analysis of the IEEE 802.11 Distributed Coordination Function,' IEEE Journal on Selected Areas in Communications, vol. 18, no. 3, pp. 535-547, 2000.

[10] G. Bianchi and I. Tinnirello, "Remarks on IEEE 802.11 DCF Performance Analysis," IEEE Communications Letters, vol. 9, no. 8, pp. 765767, 2005

[11] E.-S. Jung and N. H. Vaidya, "An Energy Efficient MAC Protocol for Wireless LANs," in IEEE INFOCOM, vol. 3, 2002, pp. 1756-1764. 